\title{
The Gravitational Potential of Two Point Masses at Five Loops
}

\author{
Johannes Blümlein \\ Deutsches Elektronen-Synchrotron, DESY, Platanenallee 6, D-15738 Zeuthen, Germany \\ E-mail: Johannes.Bluemlein@desy. de
}

\section{Andreas Maier*}

Deutsches Elektronen-Synchrotron, DESY, Platanenallee 6, D-15738 Zeuthen, Germany

E-mail: andreas.martin.maier@desy.de

\section{Peter Marquard}

Deutsches Elektronen-Synchrotron, DESY, Platanenallee 6, D-15738 Zeuthen, Germany

E-mail: Peter.Marquardedesy.de

\begin{abstract}
Corrections to the Newtonian gravitational potential from general relativity can be derived in a combined expansion around flat spacetime and a small velocity of the interacting bodies. We present the calculation of the static five-loop corrections in an effective field theory framework using techniques from multi-loop computations in particle physics.
\end{abstract}

14th International Symposium on Radiative Corrections (RADCOR2019)

9-13 September 2019

Palais des Papes, Avignon, France

${ }^{*}$ Speaker. 


\section{Introduction}

Accurate wave form templates are crucial for any gravitational wave detection experiment. Significant improvements will be needed in order to match the requirements of next-generation experiments such as LISA or the Einstein telescope. The signals detected so far match the expectations for gravitational wave emission from mergers of two compact objects, such as black holes or neutron stars. Templates for this scenario are constructed with the help of the effective one-body formalism [1], which in turn relies on calculations from first principles describing the different phases of the binary system's evolution. Of particular importance is the inspiral phase, where the compact objects are well separated and the relative velocity is small compared to the speed of light.

Indeed, for a sufficiently large distance the system is described well by Newtonian mechanics. The average kinetic and gravitational energy are then related via the virial theorem, which predicts the scaling

$$
v \sim \sqrt{\frac{G m}{r}} \ll 1
$$

where $v$ is the characteristic velocity of a compact object, $m$ its mass and $r$ the distance between the two objects. $G$ denotes Newton's constant. Corrections from general relativity can then be obtained in a simultaneous expansion in the small velocity and coupling, the Post-Newtonian (PN) expansion. The $k \mathrm{PN}$ order corresponds to a suppression by $v^{2 k}$.

All relevant parameters of the system are connected by the same small expansion parameter. The system emits gravitational quadrupole waves, whose frequency is twice the orbital frequency $\omega \sim \frac{v}{r}$. The wavelength $\lambda$ is therefore of the order of $\frac{r}{v}$. On the other side of the spectrum, the size of the compact objects is characterised by the Schwarzschild radius $r_{s}=2 G m \sim r v^{2}$. In the following, we will assume point-like objects. Finite-size effects first contribute at 5PN order for neutron stars, and at 6 PN order for black holes [2]. In the events detected at LIGO and VIRGO [3] no evidence for non-zero spin has been observed so far and we will not discuss spin effects in the remainder of this note.

For conservative dynamics, the Lagrangian and Hamiltonian of an inspiraling binary system has been calculated to 4PN order in different formalisms [4-8]. First corrections at 5PN order have been obtained in [9-12]. In the following we discuss the calculation of the static 5PN interaction potential in a non-relativistic effective field theory [13].

\section{Expansion of the general relativity action}

Our starting point is the Einstein-Hilbert action in harmonic gauge in $d=3-2 \varepsilon$ spatial dimensions and one time dimension, viz.

$$
S_{\mathrm{EH}}+S_{\mathrm{GF}}=\frac{1}{16 \pi G} \int d^{d+1} x \sqrt{-g}\left(R-\frac{1}{2} \Gamma_{\mu} \Gamma^{\mu}\right),
$$

where $g$ is the determinant of the metric $g^{\mu v}, R$ the scalar curvature, and $\Gamma^{\mu}=g^{\alpha \beta} \Gamma_{\alpha \beta}^{\mu}$ with Christoffel symbols $\Gamma_{\alpha \beta}^{\mu}$. Our aim is to first perform a PN expansion of this action, which includes an expansion around the flat metric $\eta=\operatorname{diag}(-1,1,1,1)$. In this sense, the compact objects induce 
non-perturbative short-distance fluctuations which can be absorbed into the point-particle action

$$
S_{\mathrm{pp}}=-\sum_{i=1}^{2} m_{i} \int d \tau=-\sum_{i=1}^{2} m_{i} \int d t \sqrt{-g_{\mu \nu} \frac{\partial x_{i}^{\mu}}{\partial t} \frac{\partial x_{i}^{v}}{\partial t}} .
$$

This is akin to the operator product expansion in QCD, where non-perturbative field modes are absorbed into local condensates. The resulting action is

$$
S_{\mathrm{GR}}=S_{\mathrm{EH}}+S_{\mathrm{GF}}+S_{\mathrm{pp}} .
$$

To facilitate the PN expansion of this action we employ a temporal Kaluza-Klein decomposition of the metric [14]:

$$
g^{\mu v}=e^{2 \phi}\left(\begin{array}{lc}
-1 & A_{j} \\
A_{i} & e^{-2 \frac{d-1}{d-2} \phi}\left(\delta_{i j}+\sigma_{i j}\right)-A_{i} A_{j}
\end{array}\right) .
$$

The coupling of the point objects to the spatial vector field $A$ and the tensor field $\sigma$ are suppressed by $v^{1}$ and $v^{2}$, respectively. This greatly simplifies the Feynman rules contributing at a given order in the PN expansion.

\section{Effective theory matching}

In the next step, we match the expanded general relativity action of eq. (2.3) to the action of a non-relativistic effective theory of gravity (NRGR). The derivation is similar to the one of non-relativistic QCD (NRQCD) [15]. We distinguish between two relevant modes of the graviton fields $\phi, A$, and $\sigma$. Potential (or orbital) gravitons have wavelengths of the order of the orbital separation $r$. In the matching to NRGR, they are integrated out and their effects are absorbed into interaction potentials. The radiation modes (called ultrasoft in NRQCD) are associated with the emitted gravitational waves with wavelengths of the order of $\frac{r}{v}$. They remain part of the effective theory. In contrast to potential gravitons, radiation gravitons are parametrically on-shell. Both modes have frequencies of the order of $\frac{r}{v}$.

At leading order, NRGR is just Newtonian mechanics with the action

$$
S_{\mathrm{NRGR}}=\int d t \frac{1}{2} m_{1} v_{1}^{2}+\frac{1}{2} m_{2} v_{2}^{2}+\frac{G m_{1} m_{2}}{r}+\ldots,
$$

where the ellipsis denotes higher-order PN corrections to the kinetic energy, the potential, and terms including the radiation fields.

For the matching of the two theories, i.e. to determine the parameters of the effective theory, we equate the amplitudes for the scattering of the two compact objects under the exchange of a four-momentum $q$ that is negligible compared to the object masses $m_{1}, m_{2}$. On the effective theory side, the objects interact via the potential. In perturbative general relativity, the interaction is transmitted by the gravitons $\phi, A, \sigma$ in the potential region. The matching equation then reads

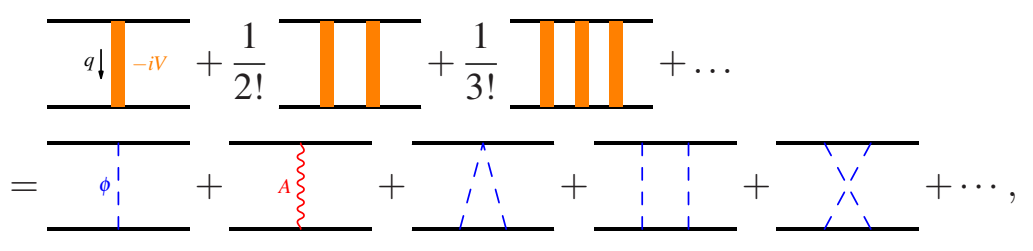


where the solid black lines illustrate the classical point object sources. Diagrams with closed graviton loops correspond to quantum corrections. These are highly suppressed and can be safely neglected.

Adding unity and taking the logarithm on both sides of the matching equation (3.2) eliminates all higher iterations of the potential insertion in the effective theory and the graviton exchange diagrams that are reducible when cutting all source lines, for example the box and crossed-box diagrams shown in (3.2). The reason for this is the following [16]. In position space, we label each interaction vertex $V$ with a time $t_{V}$ and integrate over all time variables. Initially, the vertices along a given source line in each diagram are ordered in time. That is, a source line connecting two vertices 1 and 2 numbered from left to right corresponds to a step function $\Theta\left(t_{2}-t_{1}\right)$. These step functions can be eliminated by adding up all diagrams obtained by permuting all vertices along each source line. After removing all step functions any source-reducible diagrams factorises and cancels against the product of lower-order diagrams when taking the logarithm.

As an example, let us consider the one-loop seagull diagram. Indicating the time ordering with an arrow along the source line we can write

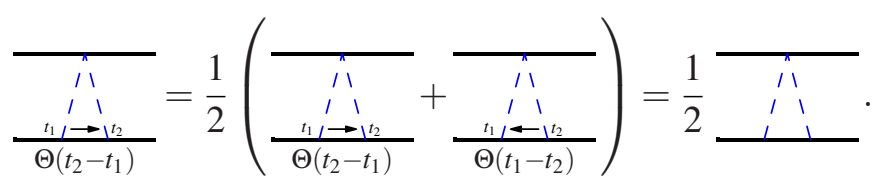

In the second step we have exploited the symmetry of the diagram under exchange of the vertices on the lower source line. Applying the same procedure to the sum of a one-loop box and the corresponding crossed-box diagram we obtain

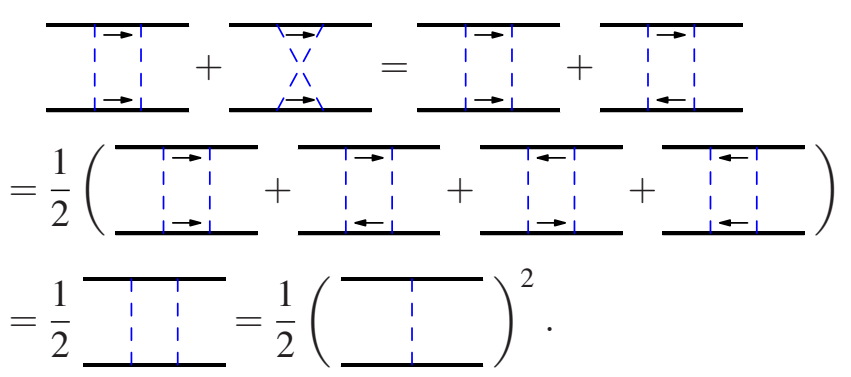

The factorisation in the last step is evident in position space. The resulting product is cancelled against the contribution from the square of the tree-level scalar exchange diagram upon taking the logarithm.

In the following, we restrict ourselves to the static limit $v_{1}=v_{2}=0$. In this case, the sources only couple directly to the scalar $\phi$. Vector gravitons $A$ are then always produced in pairs and are therefore always part of pure graviton loops. As mentioned before, pure graviton loops do not contribute in the classical limit and the $A$ field decouples completely from the theory.

\section{Setup of the calculation}

The diagrams contributing to the static gravitational potential can be calculated using standard multi-loop tools and techniques. We employ QGRAF [17] to generate FORM [18, 19] code for the diagrams. We eliminate diagrams that are irrelevant according to the criteria listed in Section 3. 
Since the only scale is given by the external momentum $q$ the diagrams belong to the well-studied class of massless propagators. At five-loop order, we identify 22 topologies, see [11].

In the next step, we perform the symmetrisation discussed in Section 3 and insert the momentumspace Feynman rules obtained from the expanded general relativity action, cf. Section 2:

$$
\begin{aligned}
& =-\frac{i}{2 c_{d} \vec{p}^{2}}
\end{aligned}
$$

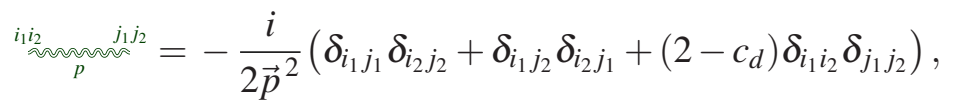

$$
\begin{aligned}
& \frac{m_{i}}{\stackrel{\vdots}{n}}=-i \frac{m_{i}}{m_{\mathrm{Pl}}^{n}}, \\
& i_{p_{2}}^{p_{1}}=i \frac{c_{d}}{2 m_{\mathrm{Pl}}}\left(V_{\phi \phi \sigma}^{i_{1} i_{2}}+V_{\phi \phi \sigma}^{i_{2} i_{1}}\right), \\
& V_{\phi \phi \sigma}^{i_{1} i_{2}}=\vec{p}_{1} \cdot \vec{p}_{2} \delta^{i_{1} i_{2}}-2 p_{1}^{i_{1}} p_{2}^{i_{2}}
\end{aligned}
$$

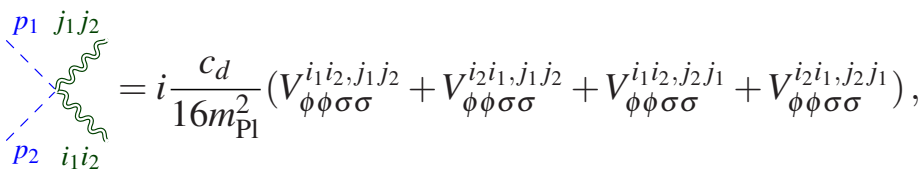

$$
\begin{aligned}
& V_{\phi \phi \sigma \sigma}^{i_{1} i_{2}, j_{1} j_{2}}=\vec{p}_{1} \cdot \vec{p}_{2}\left(\delta^{i_{1} i_{2}} \delta^{j_{1} j_{2}}-2 \delta^{i_{1} j_{1}} \delta^{i_{2} j_{2}}\right)-2\left(p_{1}^{i_{1}} p_{2}^{i_{2}} \delta^{j_{1} j_{2}}+p_{1}^{j_{1}} p_{2}^{j_{2}} \delta^{i_{1} i_{2}}\right)+8 \delta^{i_{1} j_{1}} p_{1}^{i_{2}} p_{2}^{j_{2}},
\end{aligned}
$$

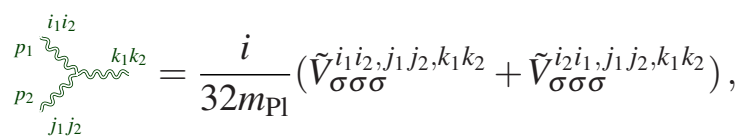

$$
\begin{aligned}
& \tilde{V}_{\sigma \sigma \sigma}^{i_{1} i_{2}, j_{1} j_{2}, k_{1} k_{2}}=V_{\sigma \sigma \sigma}^{i_{1} i_{2}, j_{1} j_{2}, k_{1} k_{2}}+V_{\sigma \sigma \sigma}^{i_{1} i_{2}, j_{2} j_{1}, k_{1} k_{2}}+V_{\sigma \sigma \sigma}^{i_{1} i_{2}, j_{1} j_{2}, k_{2} k_{1}}+V_{\sigma \sigma \sigma}^{i_{1} i_{2}, j_{2} j_{1}, k_{2} k_{1}}, \\
& V_{\sigma \sigma \sigma}^{i_{1} i_{2}, j_{1} j_{2}, k_{1} k_{2}}=\left(\vec{p}_{1}^{2}+\vec{p}_{1} \cdot \vec{p}_{2}+\vec{p}_{2}^{2}\right)\left(-\delta^{j_{1} j_{2}}\left(2 \delta^{i_{1} k_{1}} \delta^{i_{2} k_{2}}-\delta^{i_{1} i_{2}} \delta^{k_{1} k_{2}}\right)\right. \\
& \left.+2\left[\delta^{i_{1} j_{1}}\left(4 \delta^{i_{2} k_{1}} \delta^{j_{2} k_{2}}-\delta^{i_{2} j_{2}} \boldsymbol{\delta}^{k_{1} k_{2}}\right)-\delta^{i_{1} i_{2}} \boldsymbol{\delta}^{j_{1} k_{1}} \boldsymbol{\delta}^{j_{2} k_{2}}\right]\right) \\
& +2\left\{4\left(p_{1}^{k_{2}} p_{2}^{i_{2}}-p_{1}^{i_{2}} p_{2}^{k_{2}}\right) \delta^{i_{1} j_{1}} \delta^{j_{2} k_{1}}\right. \\
& +2\left[\left(p_{1}^{i_{1}}+p_{2}^{i_{1}}\right) p_{2}^{i_{2}} \delta^{j_{1} k_{1}} \delta^{j_{2} k_{2}}-p_{1}^{k_{1}} p_{2}^{k_{2}} \delta^{i_{1} j_{1}} \delta^{i_{2} j_{2}}\right] \\
& +\delta^{j_{1} j_{2}}\left[p_{1}^{k_{1}} p_{2}^{k_{2}} \delta^{i_{1} i_{2}}+2\left(p_{1}^{k_{2}} p_{2}^{i_{2}}-p_{1}^{i_{2}} p_{2}^{k_{2}}\right) \delta^{i_{1} k_{1}}-\left(p_{1}^{i_{1}}+p_{2}^{i_{1}}\right) p_{2}^{i_{2}} \delta^{k_{1} k_{2}}\right] \\
& +p_{2}^{j_{2}}\left(4 p_{1}^{i_{2}} \delta^{i_{1} k_{1}} \delta^{j_{1} k_{2}}+p_{1}^{j_{1}}\left(2 \delta^{i_{1} k_{1}} \delta^{i_{2} k_{2}}-\delta^{i_{1} i_{2}} \delta^{k_{1} k_{2}}\right)\right. \\
& \left.+2\left[\delta^{i_{1} j_{1}}\left(p_{1}^{i_{2}} \delta^{k_{1} k_{2}}-2 p_{1}^{k_{2}} \delta^{i_{2} k_{1}}\right)-p_{1}^{k_{2}} \delta^{i_{1} i_{2}} \delta^{j_{1} k_{1}}\right]\right) \\
& +p_{1}^{j_{2}}\left(p_{1}^{j_{1}}\left(2 \delta^{i_{1} k_{1}} \delta^{i_{2} k_{2}}-\delta^{i_{1} i_{2}} \delta^{k_{1} k_{2}}\right)-4 p_{2}^{i_{2}} \delta^{i_{1} k_{1}} \delta^{j_{1} k_{2}}\right. \\
& \left.\left.+2\left[p_{2}^{k_{2}} \delta^{i_{1} i_{2}} \delta^{j_{1} k_{1}}+\delta^{i_{1} j_{1}}\left(2 p_{2}^{k_{2}} \delta^{i_{2} k_{1}}-p_{2}^{i_{2}} \delta^{k_{1} k_{2}}\right)\right]\right)\right\},
\end{aligned}
$$

with $c_{d}=2 \frac{d-1}{d-2}, m_{\mathrm{Pl}}=1 / \sqrt{32 \pi G}$. There is no propagator associated with the classical sources. We perform an expansion around $\varepsilon=\frac{3-d}{2} \rightarrow 0$ and exploit integration-by-parts identities [20] to reduce the scalar integrals to a small set of master integrals. The reduction is based on Laporta's algorithm [21], implemented in our in-house code crusher [22]. We find that at five loops only four out of eight master integrals contribute in the limit $\varepsilon \rightarrow 0$. Similar to the findings of [10], we 
observe that all of them factorise into lower-order integrals. Defining $L$-loop master integrals with propagator momenta $\vec{p}_{1}, \ldots, \vec{p}_{P}$ as

$$
M_{P}=\int\left(\prod_{i=1}^{L} \frac{d^{d} l_{i}}{\pi^{d / 2}}\right) \frac{1}{\vec{p}_{1}^{2} \ldots \vec{p}_{P}^{2}}
$$

we obtain

$$
\begin{aligned}
& =\frac{\Gamma\left(6-\frac{5 d}{2}\right) \Gamma^{6}\left(-1+\frac{d}{2}\right)}{\Gamma(-6+3 d)}, \\
& =\frac{\Gamma\left(7-\frac{5 d}{2}\right) \Gamma(3-d) \Gamma\left(2-\frac{d}{2}\right) \Gamma^{7}\left(-1+\frac{d}{2}\right) \Gamma(5-2 d)}{\Gamma\left(5-\frac{3}{2} d\right) \Gamma(-2+d) \Gamma\left(-3+\frac{3}{2} d\right) \Gamma(-7+3 d)} \\
& = \\
& =-8 \pi^{7 / 2} S_{\varepsilon}^{5}\left[\frac{2}{\varepsilon}-4(1-\ln (2))-\left(48+8 \ln (2)-4 \ln ^{2}(2)-105 \zeta_{2}\right) \varepsilon+(480-96 \ln (2)\right. \\
& \\
& \left.\left.=\frac{8}{2}(2) \ln ^{3}(2)-530 \zeta_{2}+402 \ln (2) \zeta_{2}-\frac{1522}{3} \zeta_{3}\right) \varepsilon^{2}\right]+O\left(\varepsilon^{3}\right)
\end{aligned}
$$

where $S_{\varepsilon}=\exp \left(-\gamma_{E} \varepsilon\right)$. The first three diagrams can be decomposed into products of one-loop integrals

$$
=\frac{1}{a}=\frac{\Gamma\left(\frac{d}{2}-a\right) \Gamma\left(\frac{d}{2}-b\right) \Gamma\left(a+b-\frac{d}{2}\right)}{\Gamma(a) \Gamma(b) \Gamma(d-a-b)},
$$

whereas the last master integral also contains a factor of

$$
\Longrightarrow=2 \pi^{2} S_{\varepsilon}^{4}\left[\frac{1}{\varepsilon^{2}}+\frac{2}{\varepsilon}-2\left(16-\zeta_{2}\right)+16\left[9-6 \zeta_{2}\left(\frac{13}{8}-\ln (2)\right)-\frac{77}{6} \zeta_{3}\right]\right] \varepsilon+O\left(\varepsilon^{2}\right)
$$

which was calculated in $[23,24]$.

Up to four loops, our results for the potential agree with the previous calculations in the effective field theory framework $[6,13,25,26]$. At five loops we obtain

$$
V_{5 \mathrm{PN}}^{S}=\frac{G^{6}}{r^{6}} m_{1} m_{2}\left[\frac{5}{16}\left(m_{1}^{5}+m_{2}^{5}\right)+\frac{91}{6} m_{1} m_{2}\left(m_{1}^{3}+m_{2}^{3}\right)+\frac{653}{6} m_{1}^{2} m_{2}^{2}\left(m_{1}+m_{2}\right)\right],
$$

in agreement with a concurrent independent calculation [10].

\section{Acknowledgements}

This project has received funding from the European Union's Horizon 2020 research and innovation programme under the Marie Skłodowska-Curie grant agreement No. 764850, SAGEX, and COST action CA16201: Unraveling new physics at the LHC through the precision frontier. 


\section{References}

[1] A. Buonanno and T. Damour, Effective one-body approach to general relativistic two-body dynamics, Phys. Rev. D59 (1999) 084006 [gr-qc/9811091].

[2] T. Damour, Gravitational radiation and the motion of compact bodies., vol. 124, pp. 59-144. 1983.

[3] LIGO ScIENTIFIC, VIRGO collaboration, GWTC-1: A Gravitational-Wave Transient Catalog of Compact Binary Mergers Observed by LIGO and Virgo during the First and Second Observing Runs, Phys. Rev. X9 (2019) 031040 [1811.12907].

[4] T. Damour, P. Jaranowski and G. Schäfer, Conservative dynamics of two-body systems at the fourth post-Newtonian approximation of general relativity, Phys. Rev. D93 (2016) 084014 [1601.01283].

[5] L. Bernard, L. Blanchet, A. Bohé, G. Faye and S. Marsat, Energy and periastron advance of compact binaries on circular orbits at the fourth post-Newtonian order, Phys. Rev. D95 (2017) 044026 [1610.07934].

[6] S. Foffa, P. Mastrolia, R. Sturani and C. Sturm, Effective field theory approach to the gravitational two-body dynamics, at fourth post-Newtonian order and quintic in the Newton constant, Phys. Rev. $\mathbf{D 9 5}$ (2017) 104009 [1612.00482].

[7] S. Foffa and R. Sturani, Conservative dynamics of binary systems to fourth Post-Newtonian order in the EFT approach I: Regularized Lagrangian, Phys. Rev. D100 (2019) 024047 [1903. 05113 ].

[8] S. Foffa, R. A. Porto, I. Rothstein and R. Sturani, Conservative dynamics of binary systems to fourth Post-Newtonian order in the EFT approach II: Renormalized Lagrangian, Phys. Rev. D100 (2019) 024048 [1903.05118].

[9] Z. Bern, C. Cheung, R. Roiban, C.-H. Shen, M. P. Solon and M. Zeng, Scattering Amplitudes and the Conservative Hamiltonian for Binary Systems at Third Post-Minkowskian Order, Phys. Rev. Lett. 122 (2019) 201603 [1901.04424].

[10] S. Foffa, P. Mastrolia, R. Sturani, C. Sturm and W. J. Torres Bobadilla, Static two-body potential at fifth post-Newtonian order, Phys. Rev. Lett. 122 (2019) 241605 [1902.10571].

[11] J. Blümlein, A. Maier and P. Marquard, Five-Loop Static Contribution to the Gravitational Interaction Potential of Two Point Masses, Phys. Lett. B800 (2020) 135100 [1902.11180].

[12] D. Bini, T. Damour and A. Geralico, Novel approach to binary dynamics: application to the fifth post-Newtonian level, 1909.02375.

[13] W. D. Goldberger and I. Z. Rothstein, An Effective field theory of gravity for extended objects, Phys. Rev. D73 (2006) 104029 [hep-th/ 0409156$].$

[14] B. Kol and M. Smolkin, Einstein's action and the harmonic gauge in terms of Newtonian fields, Phys. Rev. D85 (2012) 044029 [1009.1876].

[15] W. E. Caswell and G. P. Lepage, Effective Lagrangians for Bound State Problems in QED, QCD, and Other Field Theories, Phys. Lett. 167B (1986) 437.

[16] W. Fischler, Quark - anti-Quark Potential in QCD, Nucl. Phys. B129 (1977) 157.

[17] P. Nogueira, Automatic Feynman graph generation, J. Comput. Phys. 105 (1993) 279.

[18] J. A. M. Vermaseren, New features of FORM, math-ph/0010025.

[19] M. Tentyukov and J. A. M. Vermaseren, The Multithreaded version of FORM, Comput. Phys. Commun. 181 (2010) 1419 [hep-ph/0 702279]. 
[20] K. G. Chetyrkin and F. V. Tkachov, Integration by Parts: The Algorithm to Calculate beta Functions in 4 Loops, Nucl. Phys. B192 (1981) 159.

[21] S. Laporta, High precision calculation of multiloop Feynman integrals by difference equations, Int. J. Mod. Phys. A15 (2000) 5087 [hep-ph/ 0102033$].$

[22] P. Marquard and D. Seidel, Crusher. Unpublished.

[23] R. N. Lee and K. T. Mingulov, Introducing SummerTime: a package for high-precision computation of sums appearing in DRA method, Comput. Phys. Commun. 203 (2016) 255 [1507. 04256 ].

[24] T. Damour and P. Jaranowski, Four-loop static contribution to the gravitational interaction potential of two point masses, Phys. Rev. D95 (2017) 084005 [1701.02645].

[25] J. B. Gilmore and A. Ross, Effective field theory calculation of second post-Newtonian binary dynamics, Phys. Rev. D78 (2008) 124021 [0810.1328].

[26] S. Foffa and R. Sturani, Effective field theory calculation of conservative binary dynamics at third post-Newtonian order, Phys. Rev. D84 (2011) 044031 [1104.1122]. 